Supporting Information

\title{
Differential aggregation properties of mutant human and bovine rhodopsin
}

\author{
Sreelakshmi Vasudevan and Paul S.-H. Park \\ Department of Ophthalmology and Visual Sciences, Case Western Reserve University, \\ Cleveland, $\mathrm{OH} 44106$
}



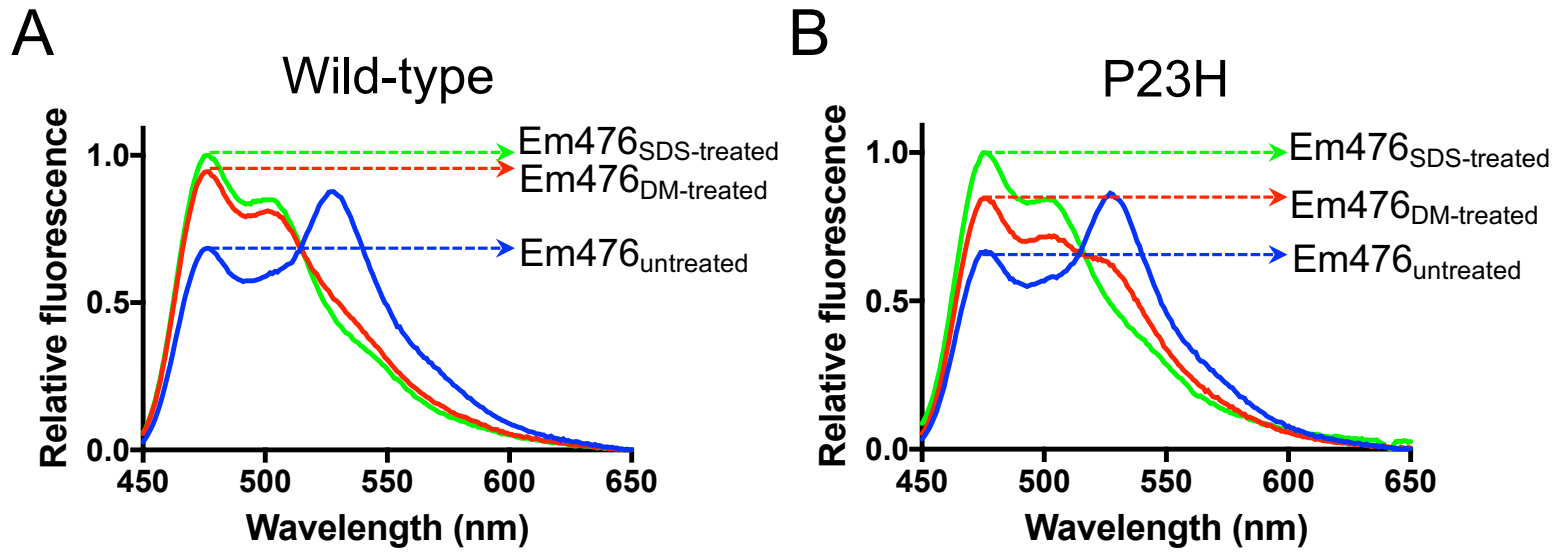

Figure S1. Examples of fluorescence emission spectra. Fluorescence emission spectra were obtained from cells coexpressing mTq2- and YFP-tagged bovine wild-type (A) or bovine $\mathrm{P} 23 \mathrm{H}$ (B) rhodopsin after excitation at $425 \mathrm{~nm}$. Emission spectra were obtained on untreated cells (blue), cells treated with DM (red) and then SDS (green). The fluorescence emission signal at $476 \mathrm{~nm}$ is used to compute the FRET efficiency, as described in the Materials and Methods. 
Table S1. Human rhodopsin FRET curve analysis

\begin{tabular}{|c|c|c|c|c|c|c|}
\hline \multirow[b]{3}{*}{$\begin{array}{c}\text { Coexpressed } \\
\text { receptors and } \\
\text { treatment } \\
\end{array}$} & \multicolumn{6}{|c|}{ Parameter } \\
\hline & \multicolumn{3}{|c|}{$E_{\max }$} & \multicolumn{3}{|c|}{ EC $_{50}$} \\
\hline & Total & $\begin{array}{c}\text { DM- } \\
\text { sensitive }\end{array}$ & $\begin{array}{c}\text { DM- } \\
\text { insensitive }\end{array}$ & Total & $\begin{array}{c}\text { DM- } \\
\text { sensitive }\end{array}$ & $\begin{array}{c}\text { DM- } \\
\text { insensitive }\end{array}$ \\
\hline $\begin{array}{l}\text { WT-YFP + } \\
\text { WT-mTq2 }\end{array}$ & $0.47 \pm 0.04$ & $0.41 \pm 0.03$ & $0.07 \pm 0.01$ & $1.14 \pm 0.43$ & $1.48 \pm 0.51$ & $0.34 \pm 0.53$ \\
\hline $\begin{array}{l}\text { P23H-YFP + } \\
\text { P23H-mTq2 }\end{array}$ & $0.47 \pm 0.03$ & $0.18 \pm 0.02$ & $0.31 \pm 0.03$ & $1.32 \pm 0.26$ & $0.85 \pm 0.31$ & $1.83 \pm 0.38$ \\
\hline $\begin{array}{c}\text { P23H-YFP + } \\
\text { P23H-mTq2 } \\
+ \text { 9-cis retinal }\end{array}$ & $0.51 \pm 0.04$ & $0.31 \pm 0.03$ & $0.21 \pm 0.02$ & $0.96 \pm 0.28$ & $1.14 \pm 0.34$ & $0.77 \pm 0.30$ \\
\hline $\begin{array}{l}\text { P23H-YFP + } \\
\text { P23H-mTq2 } \\
+ \text { metformin }\end{array}$ & $0.48 \pm 0.04$ & $0.23 \pm 0.02$ & $0.26 \pm 0.02$ & $1.87 \pm 0.34$ & $1.88 \pm 0.49$ & $1.89 \pm 0.34$ \\
\hline $\begin{array}{c}\text { P23H-YFP + } \\
\text { WT-mTq2 }\end{array}$ & $0.13 \pm 0.01$ & $0.03 \pm 0.00$ & $0.10 \pm 0.01$ & $0.72 \pm 0.16$ & $0.41 \pm 0.15$ & $0.85 \pm 0.20$ \\
\hline $\begin{array}{c}\text { P23H-YFP + } \\
\text { WT-mTq2 } \\
+ \text { 9-cis retinal }\end{array}$ & $0.48 \pm 0.03$ & $0.18 \pm 0.02$ & $0.30 \pm 0.02$ & $1.70 \pm 0.24$ & $2.09 \pm 0.68$ & $1.54 \pm 0.24$ \\
\hline $\begin{array}{c}\text { P23H-YFP + } \\
\text { WT-mTq2 } \\
+ \text { metformin }\end{array}$ & $0.14 \pm 0.01$ & $0.06 \pm 0.01$ & $0.08 \pm 0.01$ & $1.89 \pm 0.38$ & $2.46 \pm 0.90$ & $1.58 \pm 0.31$ \\
\hline
\end{tabular}

Data in Figs. 2 and 7 were fit to a rectangular hyperbolic function as described in the Materials and Methods. Fitted values for the maximal FRET efficiency $\left(E_{\max }\right)$ and $\mathrm{EC}_{50}$ are shown along with the standard errors. 
Table S2. Bovine rhodopsin FRET curve analysis

\begin{tabular}{|c|c|c|c|c|c|c|}
\hline \multirow[b]{3}{*}{$\begin{array}{c}\text { Coexpressed } \\
\text { receptors and } \\
\text { treatment } \\
\end{array}$} & \multicolumn{6}{|c|}{ Parameter } \\
\hline & \multicolumn{3}{|c|}{$E_{\max }$} & \multicolumn{3}{|c|}{ EC $_{50}$} \\
\hline & Total & $\begin{array}{c}\text { DM- } \\
\text { sensitive }\end{array}$ & $\begin{array}{c}\text { DM- } \\
\text { insensitive }\end{array}$ & Total & $\begin{array}{c}\text { DM- } \\
\text { sensitive }\end{array}$ & $\begin{array}{c}\text { DM- } \\
\text { insensitive }\end{array}$ \\
\hline $\begin{array}{l}\text { WT-YFP + } \\
\text { WT-mTq2 }\end{array}$ & $0.51 \pm 0.04$ & $0.46 \pm 0.03$ & $0.07 \pm 0.01$ & $1.26 \pm 0.37$ & $1.73 \pm 0.40$ & $0.38 \pm 0.57$ \\
\hline $\begin{array}{l}\text { P23H-YFP + } \\
\text { P23H-mTq2 }\end{array}$ & $0.51 \pm 0.04$ & $0.28 \pm 0.02$ & $0.23 \pm 0.02$ & $1.62 \pm 0.36$ & $1.51 \pm 0.38$ & $1.66 \pm 0.44$ \\
\hline $\begin{array}{c}\text { P23H-YFP + } \\
\text { P23H-mTq2 } \\
+ \text { 9-cis retinal }\end{array}$ & $0.66 \pm 0.03$ & $0.36 \pm 0.03$ & $0.30 \pm 0.02$ & $1.77 \pm 0.21$ & $1.55 \pm 0.34$ & $1.92 \pm 0.38$ \\
\hline $\begin{array}{l}\text { P23H-YFP + } \\
\text { P23H-mTq2 } \\
+ \text { metformin }\end{array}$ & $0.60 \pm 0.03$ & $0.35 \pm 0.02$ & $0.25 \pm 0.01$ & $1.40 \pm 0.26$ & $1.46 \pm 0.33$ & $1.36 \pm 0.21$ \\
\hline $\begin{array}{c}\text { P23H-YFP + } \\
\text { WT-mTq2 }\end{array}$ & $0.32 \pm 0.02$ & $0.20 \pm 0.03$ & $0.12 \pm 0.01$ & $2.94 \pm 0.60$ & $4.16 \pm 1.26$ & $2.09 \pm 0.40$ \\
\hline $\begin{array}{c}\text { P23H-YFP + } \\
\text { WT-mTq2 } \\
+ \text { 9-cis retinal }\end{array}$ & $0.47 \pm 0.02$ & $0.34 \pm 0.03$ & $0.14 \pm 0.02$ & $2.58 \pm 0.32$ & $3.18 \pm 0.89$ & $1.54 \pm 0.87$ \\
\hline $\begin{array}{c}\text { P23H-YFP + } \\
\text { WT-mTq2 } \\
+ \text { metformin }\end{array}$ & $0.49 \pm 0.01$ & $0.28 \pm 0.01$ & $0.21 \pm 0.02$ & $1.13 \pm 0.09$ & $0.88 \pm 0.20$ & $1.54 \pm 0.46$ \\
\hline
\end{tabular}

Data in Figs. 2 and 7 were fit to a rectangular hyperbolic function as described in the Materials and Methods. Fitted values for the maximal FRET efficiency $\left(E_{\max }\right)$ and $\mathrm{EC}_{50}$ are shown along with the standard errors. 
Table S3. Extra sum of squares F test analysis

\begin{tabular}{|c|c|c|c|c|c|c|}
\hline \multirow[b]{3}{*}{$\begin{array}{c}\text { Coexpressed } \\
\text { receptors and } \\
\text { treatment }\end{array}$} & \multicolumn{6}{|c|}{$P$-value } \\
\hline & \multicolumn{3}{|c|}{ Human } & \multicolumn{3}{|c|}{ Bovine } \\
\hline & Total & $\begin{array}{c}\text { DM- } \\
\text { sensitive }\end{array}$ & $\begin{array}{c}\text { DM- } \\
\text { insensitive }\end{array}$ & Total & $\begin{array}{c}\text { DM- } \\
\text { sensitive }\end{array}$ & $\begin{array}{c}\text { DM- } \\
\text { insensitive }\end{array}$ \\
\hline $\begin{array}{l}\text { WT-YFP + } \\
\text { WT-mTq2 }\end{array}$ & $<0.0001$ & $<0.0001$ & 0.078 & $<0.0001$ & $<0.0001$ & 0.4088 \\
\hline $\begin{array}{l}\text { P23H-YFP + } \\
\text { P23H-mTq2 }\end{array}$ & $<0.0001$ & 0.0837 & $<0.0001$ & $<0.0001$ & 0.0034 & $<0.0001$ \\
\hline $\begin{array}{c}\text { P23H-YFP + } \\
\text { P23H-mTq2 } \\
+ \text { 9-cis retinal }\end{array}$ & $<0.0001$ & 0.0009 & $<0.0001$ & $<0.0001$ & $<0.0001$ & $<0.0001$ \\
\hline $\begin{array}{l}\text { P23H-YFP + } \\
\text { P23H-mTq2 } \\
+ \text { metformin }\end{array}$ & $<0.0001$ & 0.6156 & $<0.0001$ & $<0.0001$ & $<0.0001$ & $<0.0001$ \\
\hline $\begin{array}{c}\text { P23H-YFP + } \\
\text { WT-mTq2 }\end{array}$ & $<0.0001$ & $<0.0001$ & 0.0053 & 0.1553 & 0.7609 & $<0.0001$ \\
\hline $\begin{array}{c}\text { P23H-YFP + } \\
\text { WT-mTq2 } \\
+ \text { 9-cis retinal }\end{array}$ & $<0.0001$ & 0.2188 & $<0.0001$ & $<0.0001$ & 0.0003 & 0.0011 \\
\hline $\begin{array}{c}\text { P23H-YFP + } \\
\text { WT-mTq2 } \\
+ \text { metformin }\end{array}$ & $<0.0001$ & 0.0003 & 0.4462 & $<0.0001$ & $<0.0001$ & $<0.0001$ \\
\hline
\end{tabular}

Data in Figs. 2 and 7 were analyzed by an extra sum of squares $F$ test to test whether or not each $E_{\max }$ is different from the non-specific FRET $E_{\max }$ values of $0.28,0.21$, and 0.078 for total, DMsensitive, and DM-insensitive FRET, respectively. 
Table S4. Extra sum of squares F test analysis

\begin{tabular}{|c|c|c|c|}
\hline \multirow[b]{2}{*}{$\begin{array}{c}\text { Coexpressed receptors and } \\
\text { treatment }\end{array}$} & \multicolumn{3}{|c|}{ Human vs bovine ( $P$-value) } \\
\hline & Total & DM-sensitive & DM-insensitive \\
\hline WT-YFP + WT-mTq2 & 0.4654 & 0.3202 & 0.9147 \\
\hline P23H-YFP + P23H-mTq2 & 0.4526 & 0.0017 & 0.0215 \\
\hline $\begin{array}{c}\text { P23H-YFP + P23H-mTq2 } \\
\text { + 9-cis retinal }\end{array}$ & 0.0120 & 0.2276 & 0.0052 \\
\hline $\begin{array}{c}\text { P23H-YFP + P23H-mTq2 } \\
+ \text { metformin }\end{array}$ & 0.0539 & 0.0216 & 0.8271 \\
\hline P23H-YFP + WT-mTq2 & $<0.0001$ & 0.0292 & 0.0542 \\
\hline $\begin{array}{c}\text { P23H-YFP + WT-mTq2 } \\
\text { + 9-cis retinal }\end{array}$ & 0.8429 & 0.0103 & $<0.0001$ \\
\hline $\begin{array}{c}\text { P23H-YFP + WT-mTq2 } \\
\text { + metformin }\end{array}$ & $<0.0001$ & 0.0149 & 0.0028 \\
\hline
\end{tabular}

Data in Figs. 2 and 7 were analyzed by an extra sum of squares $\mathrm{F}$ test to compare the $E_{\max }$ of human and bovine forms of the receptor. 